\section{Embryo culture}

Methods and protocols

Gary D. Smith, Jason E. Swain, Thomas B.

Pool (eds)

Methods in molecular biology, vol. 912, 2012

Humana Press - Springer Verlag,

Heidelberg

ISBN: 978-1-61779-970-9

Page: 433; Figures: 40; €114,35

It has been a really pleasure to follow the presentation of all the topics related to the improvements occurring in human embryo culture as they are here presented. In other words, I am pretty sure that the readers will share the pleasure of going through this book and of catching the architectural relationshisps that the distinguished editors gave to the twenty-three chapters, the historical / logical sequence of the presentation which is frameworked by an introductory chapter dealing with the historical background of gamete and embryo culture that by itself is worth the cost of the book !

I appreciated so much this chapter written by Dr. Jacques Coehen (Tyho-Galileo research laboratories and reprogenetics, West Otange, NJ, USA) and Don Rieger (Research and development, LifeGlobal, LLC, Guelph, ON, Canada) since the reader can trace the efforts that along decades and decades of small improvements the whole scientific community was able to achieve and to provide to outstanding colleagues like Patrick Steptoe and Robert Edwards which lead to the birth of Louise Brown on July 25, 1978. Surely that date was not marking the achievement of the most satisfactory conditions for embryo culture as this book is illustrating so well. Two necessary conditions to achieve always better embryo cultures are practically presented in details, media composition and culture systems. This fact is quite clear at the light of the historical background presented in chapter one. Here the development of the media culture is followed starting from the Sydney Ringer saline solution (1883) worked out for the beating frog heart, the addition of sodium phospate by Maurice Tyrode (1910), the John Hammond (1949) and Wesley Whitten (1956) addition of glucose, antibiotics and bovine serum albumin to the Krebs-Ringer-Bicarbonate solution just to mention the early days of these researches that culminated decades later in media cultures optimized by the oviduct and uterine fluids and the presentation of now a day media.

Each of these historical steps is mirrored by a chapter dealing with the present day view: thus, there are chapters dealing with the influence on embryo culture exerted by salts and osmolality, energy sources and metabolism, amino acids and cellular homeostasis, macromolecules, antioxidants, growth factors, $\mathrm{pH}$ and buffers. The same logical presentation is behind the culture systems where the historical remarks on containers, dessicators, Pasteur pipettes, Petri dishes, inverted microscopes are actualized in several chapters dealing with embryo density, single, sequential or embryo co-culture, air quality, mineral oil overlay. Additional chapters cover the always hot debated topics of how the different environmental (i.e., methodologies) conditions influence gene expression, that is to say which are the epigenetic alteration of the gene expression brough about by the culture condition adopted by the operator.

The editors, and all of the authors, have to be acknowledged for this very well done book that will help researchers, clinicians and reproductive biologists to help those $10 \%$ of human kind afflicted by infertility and to rise the animal production rates, not to mention the reproduction of the endangered species.

CarloAlberto Redi University of Pavia 